\title{
Multiple primary lung cancers: clinical and genetic features
}

\author{
Yuntao Nie, Kezhong Chen, Jun Wang \\ Department of Thoracic Surgery, Peking University People's Hospital, Beijing 100044, China \\ Correspondence to: Jun Wang. Department of Thoracic Surgery, Peking University People’s Hospital, Beijing 100044, China. Email: wangjun@pkuph.edu.cn; \\ Kezhong Chen. Department of Thoracic Surgery, Peking University People’s Hospital, Beijing 100044, China. Email: mdkzchen@163.com. \\ Provenance: This is an invited article commissioned by the Section Editor Akira Hamada (Department of Cardiovascular, Thoracic and Pediatric \\ surgery, Faculty of Medicine, Yamagata University, Yamagata, Japan). \\ Response to: Fabian T. Multiple primary lung cancers. J Thorac Dis 2018;10:S3109-10.
}

Yu YC, Huang CS, Huang BS. Separate or intrapulmonary metastasis? J Thorac Dis 2018;10:S3128-30.

Submitted Nov 04, 2018. Accepted for publication Nov 27, 2018.

doi: $10.21037 /$ jtd.2018.11.130

View this article at: http://dx.doi.org/10.21037/jtd.2018.11.130

It is with great interest that we read the insightful comments by Dr. Fabian and Dr. Yu concerning our recent article in The fournal of Thoracic and Cardiovascular Surgery (1). We feel grateful for their recognition of the value of our article, and would like to respond to the issues that have been raised.

\section{Perspectives}

With the increase in detection rate, multiple primary lung cancers (MPLCs) have become a major challenge for clinicians. However, the prognosis of resected MPLCs is still unclear due to the large range of overall survival (OS) reported in different studies $(2,3)$. We agree with Dr. Fabian's comment noting that "there is variability in inclusion criteria which creates these dramatic diferences in outcomes".

The 8th edition of the IASLC staging proposed welldefined criteria for categorizing multiple lung cancers into four patterns: second primary lung cancer (SPLC), separate tumor nodule, multifocal lung cancer (MFLC), and the difuse pneumonic type (4). It is easy to identify patients with MFLC due to the presence of multiple ground-glass opacities (GGOs) on computerized tomography (CT) scan, and these patients are reported to have a 5 -year OS of virtually $100 \%(5,6)$. SPLC was defined as solid lesions having diferent histotypes or diferent appearances in the same histotype by a detailed histologic assessment (7). Since the fact that the outcomes of MFLC are much better than SPLC (8), blending these two patterns of disease together in one study undoubtedly resulted in data erroneously indicating improved survival. In our study, we categorized MPLCs following radiological findings (consolidation/ tumor ratio), and found a significantly different prognosis between them. This is one of the reasons why there has been a large difference in the prognosis of MPLCs among previous studies. Future studies must carefully distinguish between multifocal GGOs and multiple solid primary lung cancers.

Having established that patients with MPLC face more favorable surgical outcomes, the question arises as to how to choose the optimal surgical strategy for these patients. Based on the different characteristics of multiple pulmonary nodules, surgical procedures should be performed discriminately to suitable patients. Firstly, in accordance with the ACCP guidelines for patients with multiple solid tumors (8), it is reasonable to proceed with a resection of each lesion if there is no N2 nodal disease. Lobectomy of the main lesion is suitable, while a sub-lobar resection of one or both lesions may be necessary, depending on the patient's pulmonary reserve. Secondly, for patients with a solid-dominant tumor (DT) with other GGOs, it has been demonstrated in several studies that radical resection for DT is the most essential, while the decision whether the additional GGOs should be resected is based on the tumor size and location $(9,10)$. In our study, we collected a total of 35 patients with a dominant invasive lesion with multiple nodules, and the resulting Kaplan-Meier curves showed the 5 -year OS was $80.5 \%$, which further supports this strategy. Thirdly, for patients with multifocal GGOs, limited 


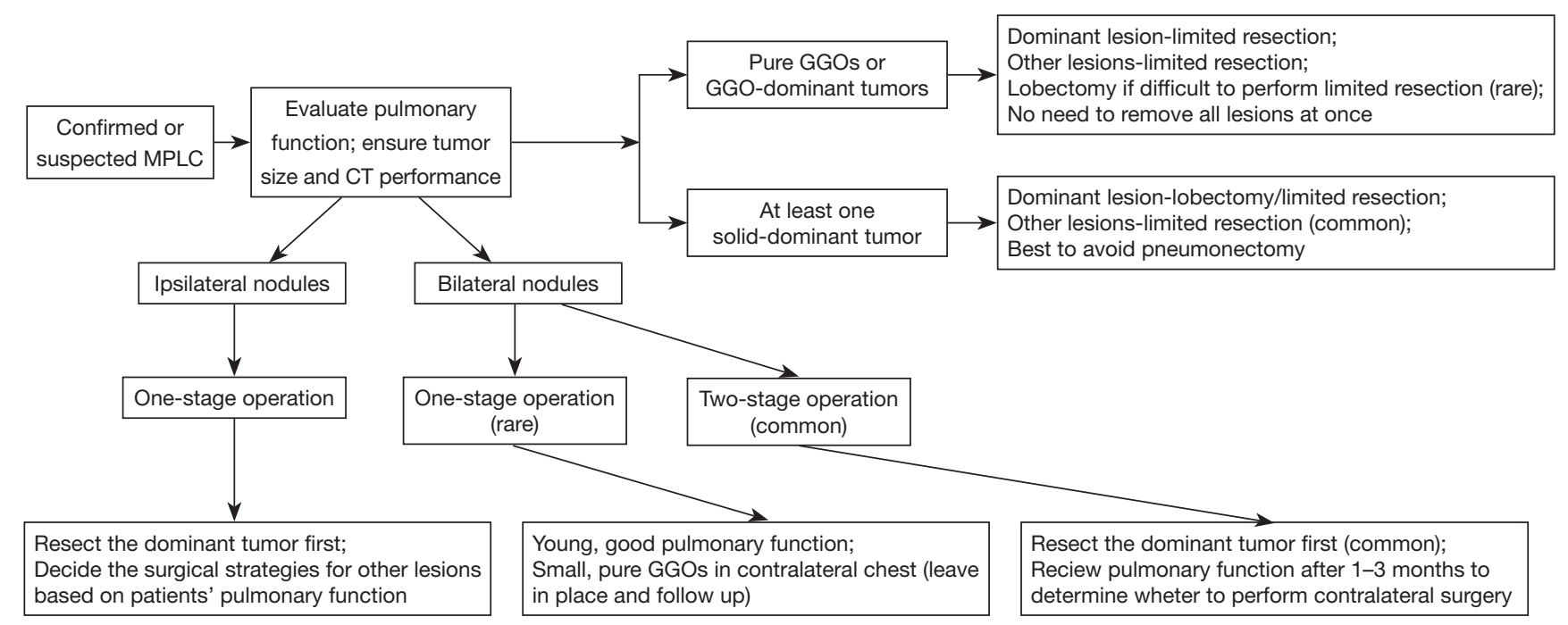

Figure 1 Treatment strategies of patients with different subgroups of multiple primary lung cancers. MPLC, multiple primary lung cancer; CT, computerized tomography; GGOs, ground-glass opacities.

resections for available lesions or leaving some of the pure GGOs unresected, were recommended by the growing data $(5,6,8)$. This recommendation was supported by the approximately $100 \%$ OS rate and the very low recurrence rate after resection of multifocal GGOs. Conclusively, we summarize the treatment strategies of patients with different subgroups of MPLCs in our own institution in Figure 1. We believe that a rigorous treatment standard for patients with MPLCs can simultaneously ensure oncologic efficiency and pulmonary reserve.

The final issue is how to differentiate between multiple primaries and metastatic disease. In a statement from the Fleischner Society and the IASLC (11,12), GGOs are considered to have independent origins, which is demonstrated in several studies by using genetic analysis in resected multiple GGOs $(1,13)$. However, it is particularly difficult to make a decision using traditional Martini and Melamed's criteria in patients with 2 solid lesions, especially when both show the same major histotype (2). Finley et al. (14) modified the criteria by adding histologic subtyping in, and considered tumors to be unrelated if different histologic subtypes were apparent. The 2013 ACCP guidelines (8) also supported the use of histologic subtyping in differentiating MPLCs and intrapulmonary metastasis. Since cumulative data has not found a worse prognosis in patients with different histologic subtypes $(3,14,15)$, we agree with the view of Dr. Fabian which endorses Finley's criteria as the basic standard to define MPLCs.
Despite Finley's criteria being able to define most of the MPLC patients, in the situation where 2 lesions having the same histologic subtype occurs (e.g., both are papillarydominant) without lymph node metastasis, it is difficult to decide appropriately. Although it was the consensus of the IASLC to advocate for performing comprehensive histologic assessment in these patients, this method is still time-consuming and based on mainly the experience of pathologists (7). It is worth mentioning that genetic analysis, to some extent, helped us distinguish between MPLCs and metastatic disease in our study. Although there never was a consensus which stated that a difference in specific mutations identifies separate primary cancers, or that mutations in the same gene define a single lineage (7), we still believe that genetic analysis will have a place in the future of diagnosis. For now, we must follow the statement in ACCP guidelines: "the data regarding molecular genetic features of cancers should be taken into account but should not be regarded as definitive by themselves or to obviate the consideration of other clinical and radiographic features" (8).

In summary, the debate on MPLCs has been going on for over decades, and still has no definite conclusion. Any information available on these patients is valuable, but needs to be treated with caution. Therefore, in accordance with the consensus of the IASLC, a careful review by a multidisciplinary tumor board that considers all available information is needed to identify and manage patients with MPLCs (7). 


\section{Acknowledgements}

None.

\section{Footnote}

Conflicts of Interest: The authors have no conflicts of interest to declare.

\section{References}

1. Chen K, Chen W, Cai J, et al. Favorable prognosis and high discrepancy of genetic features in surgical patients with multiple primary lung cancers. J Thorac Cardiovasc Surg 2018;155:371-9.e1.

2. Martini N, Melamed MR. Multiple primary lung cancers. J Thorac Cardiovasc Surg 1975;70:606-12.

3. Yu YC, Hsu PK, Yeh YC, et al. Surgical Results of Synchronous Multiple Primary Lung Cancers: Similar to the Stage-Matched Solitary Primary Lung Cancers? Ann Thorac Surg 2013;96:1966-74.

4. Detterbeck FC, Nicholson AG, Franklin WA, et al. The IASLC Lung Cancer Staging Project: Summary of Proposals for Revisions of the Classification of Lung Cancers with Multiple Pulmonary Sites of Involvement in the Forthcoming Eighth Edition of the TNM Classification. J Thorac Oncol 2016;11:639-50.

5. Kim TJ, Goo JM, Lee KW, et al. Clinical, pathological and thin-section CT features of persistent multiple groundglass opacity nodules: comparison with solitary groundglass opacity nodule. Lung Cancer 2009;64:171-8.

6. Mun M, Kohno T. Efficacy of thoracoscopic resection for multifocal bronchioloalveolar carcinoma showing pure ground-glass opacities of $20 \mathrm{~mm}$ or less in diameter. J Thorac Cardiovasc Surg 2007;134:877-82.

7. Detterbeck FC, Franklin WA, Nicholson AG, et al. The IASLC Lung Cancer Staging Project: Background Data and Proposed Criteria to Distinguish Separate Primary Lung Cancers from Metastatic Foci in Patients with Two Lung Tumors in the Forthcoming Eighth Edition of the

Cite this article as: Nie Y, Chen K, Wang J. Multiple primary lung cancers: clinical and genetic features. J Thorac Dis 2018;10(12):E832-E834. doi: 10.21037/jtd.2018.11.130
TNM Classification for Lung Cancer. J Thorac Oncol 2016;11:651-65.

8. Kozower BD, Larner JM, Detterbeck FC, et al. Special treatment issues in non-small cell lung cancer: Diagnosis and management of lung cancer, 3rd ed: American College of Chest Physicians evidence-based clinical practice guidelines. Chest 2013;143:e369-99S.

9. Gao RW, Berry MF, Kunder CA, et al. Survival and risk factors for progression after resection of the dominant tumor in multifocal, lepidic-type pulmonary adenocarcinoma. J Thorac Cardiovasc Surg 2017;154:2092-9.e2.

10. Gu B, Burt BM, Merritt RE, et al. A dominant adenocarcinoma with multifocal ground glass lesions does not behave as advanced disease. Ann Thorac Surg 2013;96:411-8.

11. Naidich DP, Bankier AA, MacMahon H, et al. Recommendations for the management of subsolid pulmonary nodules detected at CT: a statement from the Fleischner Society. Radiology 2013;266:304-17.

12. Detterbeck FC, Marom EM, Arenberg DA, et al. The IASLC Lung Cancer Staging Project: Background Data and Proposals for the Application of TNM Staging Rules to Lung Cancer Presenting as Multiple Nodules with Ground Glass or Lepidic Features or a Pneumonic Type of Involvement in the Forthcoming Eighth Edition of the TNM Classification. J Thorac Oncol 2016;11:666-80.

13. Wu C, Zhao C, Yang Y, et al. High Discrepancy of Driver Mutations in Patients with NSCLC and Synchronous Multiple Lung Ground-Glass Nodules. J Thorac Oncol 2015;10:778-83.

14. Finley DJ, Yoshizawa A, Travis W, et al. Predictors of Outcomes after Surgical Treatment of Synchronous Primary Lung Cancers. Journal Of Thoracic Oncology 2010;5:197-205.

15. Cheng H, Lei BF, Peng PJ, et al. Histologic lung cancer subtype differentiates synchronous multiple primary lung adenocarcinomas from intrapulmonary metastases. J Surg Res 2017;211:215-22. 